\title{
Effect of distractor sounds on the auditory attentional blink
}

\author{
DAWEI SHEN and TODD A. MONDOR \\ University of Manitoba, Winnipeg, Manitoba, Canada
}

\begin{abstract}
Four experiments were conducted to determine whether or not the presence and placement of distractors in a rapid serial auditory stream has any influence on the emergence of the auditory attentional blink (AB). Experiment 1 revealed that the presence of distractors is necessary to produce the auditory $\mathrm{AB}$. In Experiments 2 and 3, the auditory $\mathrm{AB}$ was reduced when the distractor immediately following the probe was replaced by silence but not when the distractor following the target was replaced by silence. Finally, in Experiment 4, only a very small auditory AB was found to remain when all distractors following the probe were replaced by silence. These results suggest that the auditory AB is affected both by the overwriting of the probe by the distractors following it and by a reduction in discriminability generated by all of the distractors presented in the sequence.
\end{abstract}

It has been established that if two targets are to be identified among distractors in a rapid serial visual presentation (RSVP) stream, correct identification of the first target may produce a deficit in the processing of the second target lasting several hundred milliseconds (see, e.g., Broadbent \& Broadbent, 1987; Chun \& Potter, 1995; Duncan, Ward, \& Shapiro, 1994; Jolicœur \& Dell' Acqua, 1998; Raymond, Shapiro, \& Arnell, 1992, 1995; Shapiro, Raymond, \& Arnell, 1994). This processing deficit, which Raymond et al. (1992) have labeled the attentional blink (AB), provides important information about the deployment of attention in the temporal domain. In most studies of the $\mathrm{AB}$, there are two principal conditions. In the experimental condition, a target (i.e., the first target) and a probe (i.e., the second target; cf. Arnell \& Jolicœur, 1999) are embedded in a rapid sequence of items and participants are required to identify both of them. In contrast, in the control condition, participants are instructed to ignore the target (if it is presented) and to identify the probe. Typically, the accuracy with which the probe is identified is higher in the control condition than in the experimental condition, and this difference decreases as the time between the target and the probe increases. This $\mathrm{AB}$ persists for approximately 400-500 msec.

Quite a large number of studies have been conducted to examine the visual $\mathrm{AB}$ phenomenon using various kinds

Part of the data from Experiment 1 was presented in a poster at the 12th Annual Meeting of the Canadian Society for Brain, Behaviour, and Cognitive Science, Vancouver, June 2002. Portions of Experiments 1, 2 , and 4 were presented in a poster at the 28th International Congress of Psychology, Beijing, August 2004. This research was supported by a grant from the Natural Sciences and Engineering Research Council of Canada to T.A.M. and by a University of Manitoba Graduate Fellowship to D.S. We thank Mari R. Jones, Sébastien Tremblay, Dylan M. Jones, and an anonymous reviewer for their helpful comments and suggestions. Correspondence concerning this article should be addressed to D. Shen, Department of Psychology, University of Manitoba, Winnipeg, MB, R3T 2N2 Canada (e-mail: umshend@cc.umanitoba.ca). of materials, such as words, letters, digits, syllables, dot patterns, keyboard symbols, line segments, geometric figures, imaginary objects, and so on (see, e.g., Broadbent \& Broadbent, 1987; Chun \& Potter, 1995; Duncan et al., 1994; Giesbrecht, Bischof, \& Kingstone, 2003; Giesbrecht \& Di Lollo, 1998; Isaak, Shapiro, \& Martin, 1999; Jolicœur, 1998; Jolicœur \& Dell'Acqua, 1998; Kawahara, Zuvic, Enns, \& Di Lollo, 2003; Kellie \& Shapiro, 2004; Potter, Staub, \& O'Connor, 2002; Raymond, 2003; Raymond et al., 1992, 1995; Shapiro et al., 1994; Ward, Duncan, \& Shapiro, 1996). Several theories have been proposed to explain the visual $\mathrm{AB}$ on the basis of these studies. Most prominent among these accounts are the attentional gate model (Raymond et al., 1992), the competition hypothesis (Shapiro et al., 1994), and the two-stage model (Chun \& Potter, 1995; Giesbrecht \& Di Lollo, 1998; Jolicœur, 1998).

According to the attentional gate model (Raymond et al., 1992), target identification is thought to require two stages. In the first stage, participants attempt to detect the target's key feature (e.g., color) after receiving instructions to search for it (e.g., "Look for a red letter"). In the second stage, the key feature is linked with the appropriate response in a process that produces conscious identification. The attentional gate model derives its name from the fact that it is based on the supposition that an attentional gate is shut and locked following detection of the critical target feature to protect the second (identification) stage from interference caused by subsequent information. Closure of the attentional gate results in active inhibition of the processing of new items, which persists for about $400 \mathrm{msec}$. Thus, detection of a probe presented during this time period is likely to be impaired.

The competition hypothesis (Shapiro et al., 1994) is based on the idea that an internal representation that includes both perceptual and conceptual characteristics is created for each item in an RSVP stream. Each successive representation is compared with an internal template de- 
fined by the instructions. When an item matches the template, it enters into visual short-term memory (VSTM), where the perceptual and conceptual characteristics of the item are integrated to form a unitary representation. Only items that enter VSTM can become response items. Both the target and the probe are admitted to VSTM. Distractor items generally have a lower probability of being admitted to VSTM because they do not match the internal template well. However, the model asserts that, relative to other distractors, items immediately following the target or the probe may be more likely to pass into VSTM because they are presented in a temporal position similar to those of the target or the probe. Because the target occurs before the probe, it is likely to be processed first. According to this theory, the $\mathrm{AB}$ is caused by competition for limited perceptual and semantic processing resources among the target, the probe, and any distractors admitted to VSTM. The competition is greatest when the probe immediately follows the target because, in this situation, these items will have the longest common processing time. Competition decreases as the time between the target and the probe increases. Thus, the probe-processing deficit is larger at shorter stimulus onset asynchronies (SOAs) between the target and the probe than at longer SOAs between the two.

A third important type of theory is based on two stages of processing. The two-stage model (Chun \& Potter, 1995), the object substitution account (Giesbrecht \& Di Lollo, 1998), and the central interference theory (Jolicœur, 1998) are all examples of this type of theory. Recently, Chun and Potter (2001) developed a model that integrates all three accounts. According to Chun and Potter (2001), in an initial rapid-detection stage, each item in the RSVP stream is momentarily detected. However, in order for the participant to successfully report the target and probe, these must be consolidated in a second, limited-capacity stage. In this stage, the target items are encoded into short-term memory (STM) in a serial fashion according to the task requirements. Thus, when the participant must consolidate both the target and the probe, there is a delay in processing the probe caused by ongoing consolidation of the preceding target. During this delay, the representation in memory of the probe may decay or be overwritten by subsequently presented distractors, and a probe processing deficit occurs. (Overwriting is a special case of interference and has emerged as the preferred label because researchers have found that in identification tasks the distractor following the probe was often mistakenly identified as the probe). The probe processing delay is greatest when the probe is presented immediately following the target. Thus, the impairment in probe processing declines as the time between the target and the probe increases.

In contrast with the substantial empirical analysis and theoretical development of the visual AB, there have been only a few investigations of the auditory AB. These investigations were focused on three kinds of materials: spoken syllables (Duncan, Martens, \& Ward, 1997; Tremblay, Vachon, \& Jones, 2005), spoken letters and digits (Arnell \& Jolicœur, 1999; Arnell \& Larson, 2002; Soto-Faraco \&
Spence, 2002), and nonverbal, nonmusical tones (Mondor, 1998). We summarize these studies below as a means of introducing both the types of experimental paradigms that have been used to study the auditory AB and the obtained results.

In Duncan et al.'s (1997) experiments, participants were presented with two concurrent auditory streams. One stream was presented in a low-pitched voice and the other in a high-pitched voice, and the target and the probe were always presented in different streams. The participants were instructed to listen for the target word "nap" or "nab" in the low-pitched stream and "cot" or "cod" in the highpitched stream. The spoken syllable "guh" was used as a distractor. SOA between successive items was $250 \mathrm{msec}$, and the interstimulus interval (ISI) was $100 \mathrm{msec}$. One stream, chosen at random on each trial, began $125 \mathrm{msec}$ before the other to increase the likelihood that the participants would perceive two separate channels. The results revealed that when the participants were instructed to listen for targets in both streams, there was a deficit in identifying the second target sound presented, whose magnitude decreased as the time between the first and second targets increased. In contrast, when the participants were instructed to listen only for targets in one stream, the presence of a preceding target in the other stream had no effect on detection accuracy. Thus, these results revealed a significant auditory $\mathrm{AB}$ for spoken verbal material. Tremblay et al. (2005) used materials similar to those of Duncan et al. (1997) and also observed an auditory AB. In addition, however, they found that the magnitude of the auditory AB increased as the heterogeneity of the distractors increased.

Arnell and Jolicœur (1999) presented their participants with a single stream of spoken digits and letters. The target was a specific spoken digit (" 1, , " 2, ," " 3 ," or " 4 "), the probe was the spoken letter "X," and the distractors were other spoken letters. Arnell and Jolicœur reported a deficit in detecting the probe if participants were also required to identify the preceding target. Interestingly, this auditory $\mathrm{AB}$ was apparent if the sequence was presented rapidly (SOAs of 105 and $120 \mathrm{msec}$ ) but not for slower sequences (135- and 150-msec SOAs). Arnell and Larson (2002) used a different technique, in which a single sequence of spoken letters was presented to the right ear. On each trial, one or two tones were presented in the left ear and participants were required to identify the letter presented simultaneously with a tone. The results revealed that identification of a second (probe) letter was impaired when it was presented immediately following a target letter. In addition, Soto-Faraco and Spence (2002) used digits as the target and the probe, and English letters as the distractors. The participants were required to identify the two targets. The results also showed a robust auditory AB.

Mondor (1998) presented listeners with a rapid sequence of pure tones. The target was a pure tone of an especially high frequency $(4000 \mathrm{~Hz})$, the probe was a complex sound comprised of multiple frequency components, and the distractors were pure tones from 452 to $3462 \mathrm{~Hz}$ in frequency. Mondor reported a robust auditory AB when 
both the target and the probe were to be detected. Furthermore, he found that the magnitude of the auditory $\mathrm{AB}$ was unchanged when the item immediately following the target was replaced by a silent period.

Evidence of an auditory $\mathrm{AB}$ has, then, been reported several times. However, the mechanism responsible for the effect remains unclear. In the theories of the visual $\mathrm{AB}$, distractors play a very important role. For example, the two-stage model (Chun \& Potter, 1995; Giesbrecht \& Di Lollo, 1998; Jolicœur, 1998) is founded on the notion that processing of the probe must await completion of target processing. The target and the distractor immediately following it may be processed together, and this can contribute to the delay in probe processing. During this delay, the probe representation may decay or be overwritten by the distractors that follow the probe, thereby generating an AB. In contrast, the competition hypothesis (Shapiro et al., 1994) rests on the assumption that competition for resources among the target, the probe, and the distractors immediately following each of them causes the visual AB. Finally, the attentional gate model (Raymond et al., 1992) suggests that the distractor immediately following the target initiates an inhibition mechanism, and this produces the visual AB. Clearly, although markedly different in most respects, all three of these theoretical accounts of the visual $\mathrm{AB}$ recognize the importance of distractors. The present study was conducted to examine the role that distractors play in producing the auditory $\mathrm{AB}$ as a means of moving toward a theoretical account of it.

\section{EXPERIMENT 1}

In Experiment 1, we examined whether or not an auditory $\mathrm{AB}$ may be obtained in the complete absence of distractor sounds. Listeners were presented with sequences consisting of either 2 or 16 sounds. Tremblay et al. (2005) recently reported that distractors were necessary to produce an auditory $\mathrm{AB}$ with spoken syllables. The experiment allowed us to determine whether or not the effect found by Tremblay et al. may characterize the auditory $\mathrm{AB}$ for nonverbal sounds.

The design of the present experiment is similar to that of some visual AB studies (e.g., Isaak et al., 1999; Jiang $\&$ Chun, 2001). Two experimental and two control conditions were used for both the 16-sound and the 2-sound sequences. In the experimental conditions, the target was always presented and participants were instructed either to listen for it or to ignore it. In the control conditions, the target was not presented and participants were instructed either to listen for it or to ignore it. This design allowed us to examine the extent to which any obtained $A B$ deficit may be caused by low-level, sensory masking or by an attentional limitation. Any AB deficit caused by sensory masking should be apparent whether the target is ignored or not (see, e.g., Shapiro et al., 1994). In contrast, an AB deficit caused by an attentional limitation should, at the very least, be larger in magnitude when the target is attended than when the target is ignored.

\section{Method}

\section{Participants}

Twenty undergraduate students enrolled in an introductory psychology course at the University of Manitoba participated in the experiment in exchange for course credit. All of the participants reported having normal hearing.

\section{Materials}

Computer and sound system. The experiment was conducted using a Dell Pentium computer running the E-Prime Software System (Psychology Software Tools, Version 1.1). Sounds were synthesized using Cool Edit 2000 (Syntrillium Software) at a sampling rate of $44100 \mathrm{~Hz}$. All sounds were $30 \mathrm{msec}$ in duration and included 2-msec linear onset/offset amplitude ramps to eliminate the onset/ offset clicks. Sounds were presented at a comfortable intensity of about 70 dB SPL from a Harman/Kardon HK-195 speaker positioned directly in front of the listeners.

Sounds. Twenty-one pure tones were used as distractors. The frequencies of these tones were log related and ranged from 529 to $1330 \mathrm{~Hz}$. The specific frequencies were 529, 554, 580, 607, 636, $666,697,730,764,800,838,877,918,961,1006,1056,1106$, $1158,1213,1270$, and $1330 \mathrm{~Hz}$. The target was composed of six 5 -msec pulses, and its frequency could be any of the 21 frequencies of the distractors. The probe was a glide that changed smoothly in frequency from 636 to $1006 \mathrm{~Hz}$.

\section{Procedure}

In all conditions, the target was presented on $50 \%$ of the trials and the probe was presented on $50 \%$ of the trials. This arrangement meant that, on different trials, target and probe were both presented; the target was presented alone; the probe was presented alone; and neither the target nor the probe was presented. Thus, there were four types of trials, all occurring equally often. On trials on which the target or the probe was not presented, a distractor was presented in its place.

On full-stream trials, there were 16 sounds in each sequence. The target could occur following either 4 or 6 distractor sounds, and the probe could occur in any of the first eight temporal positions following the target. All sounds were $30 \mathrm{msec}$ in duration, and there was a 50 -msec silent interval between successive tones. On no-stream trials, only 2 sounds were presented on each trial. The target was presented after either 400 or $560 \mathrm{msec}$ of silence (equivalent to the time period prior to target presentation when a full-stream sequence was presented), and the probe could occur $80,160,240,320,400,480$, 560 , or $640 \mathrm{msec}$ following the target (delays equivalent to those of the full-stream trials). Full-stream and no-stream trials were presented in different blocks. (We borrowed the labels no stream and full stream from the study of Isaak et al. [1999]. However, in their study the no-stream condition included the target, the item immediately following the target, the probe, and the item immediately following the probe sequences. In the present study, of course, the no-stream condition included only the target and the probe.)

There were two blocks of trials in each of the no-stream and fullstream conditions. Participants were instructed to listen for both the target and the probe in one block of trials, and listen only for the probe in the other block. The participants were required to make separate target and probe detection judgments in response to questions that appeared on the computer screen. The first question (Was the target present [press 1] or absent [press 0]?) appeared immediately after the end of each sequence, and the second question (Was the probe present [press 1] or absent [press 0]?) appeared immediately following an acceptable response to the first question. Only the second question was presented when the participants were instructed to attend only to the probe. The total time from the beginning of a trial to the appearance of the first question was the same in the no-stream and full-stream sequences.

In each of block of trials, there were 32 practice trials and 256 experimental trials. Each participant was required to complete all 
four blocks, and a Latin square was used to balance the order of the blocks. In the practice trials, each participant was required to meet a criterion of $60 \%$ correct on the probe judgments before beginning the experimental trials. All 20 participants met this criterion after a single block of practice trials.

\section{Data Analysis}

All ANOVAs reported in this article were subjected to the Greenhouse-Geisser adjustment test, because it is robust with respect to the sphericity assumption. Similarly, all tests of simple effects were performed using Bonferroni's adjustment method, and $\alpha_{\mathrm{FW}}=.05$ (this is an appropriate method to control the $\alpha_{\mathrm{FW}}$ level; see Maxwell \& Delaney, 2004, p. 581).

\section{Results}

\section{Target Detection}

Overall, the participants found the target quite easy to detect. The probability of a correct response on the target task when the target was attended was $.938(S E=.015)$. The probability of a correct response on a target-present trial (hit) was .939 , and the probability of an incorrect response on a target-absent trial (false alarm) was .063. Thus, average $d^{\prime}$ was 3.08.

A 2 (no stream vs. full stream) $\times 2$ (probe presence) within-subjects ANOVA was conducted using as the dependent variable the probability of accurate target detection when the target was present. The analysis did not reveal any significant effect (all $p s>.20$ ). Thus, the accuracy of target detection did not depend on the presence of the probe and did not differ between full-stream and no-stream sequences.

We also examined whether or not performance on the target detection task may have been influenced by the position of the probe. A 2 (no stream vs. full stream) $\times 8$ (Probe Positions +1 through +8 ) within-subjects ANOVA was conducted using as the dependent variable the probability of accurate target detection when both the target and the probe were presented. The analysis did not reveal any significant effects (all $p s>.20$ ). Thus, the accuracy of target detection was unaffected by temporal position of the probe.

\section{Probe Detection}

Overall probe detection accuracy for trials on which there was a correct response to the target was also high $(.928, S E=.015)$. The probability of a correct response on a probe-present trial (hit) was .933, and the probability of an incorrect response on a probe-absent trial (false alarm) was .077. Thus, average $d^{\prime}$ was 2.92. Clearly, therefore, the participants found the probe quite easy to detect as well.

A 2 (target judgment required vs. not required) $\times 2$ (no stream vs. full stream) $\times 2$ (target presence) $\times 8$ (Probe Positions +1 through +8 ) within-subjects ANOVA was conducted using as the dependent variable the conditional probability of accurate probe detection given a correct target detection response. This analysis revealed significant main effects of stream $[F(1,19)=15.015, p<.01]$, target presence $[F(1,19)=33.038, p<.001]$, and probe position $[F(7,133)=23.585, p<.001]$. The main effect of target judgment did not reach significance $[F(1,19)=$ 2.398, $p=.14]$.
Two-way interactions between target judgment and target presence $[F(1,19)=14.352, p<.01]$, stream and target presence $[F(1,19)=9.913, p<.01]$, target presence and probe position $[F(7,133)=16.703, p<.001]$, target judgment and probe position $[F(7,133)=2.826, p<.05]$, and stream and probe position $[F(7,133)=5.053, p<$ $.01]$ were all statistically significant. Of more importance, however, is the fact that there was a significant three-way interaction between stream, target presence, and probe position $[F(7,133)=10.155, p<.001]$ and a marginally significant interaction between target judgment, target presence, and probe position $[F(7,133)=2.305, p=.07]$. None of the other interactions reached significance (all other $p \mathrm{~s}>.10$ ).

To evaluate the first of these three-way interactions, we examined whether the difference in probe processing accuracy between the target-present and the target-absent conditions was affected by different probe positions for the no-stream and full-stream sequences separately. This analysis revealed that the main effect of target presence was significant for the full-stream $[F(1,19)=30.876$, $p<.001]$ and the no-stream $[F(1,19)=5.187, p<.05]$ sequences. The main effect of probe position was also significant for both the full-stream $[F(7,133)=17.155$, $p<.001]$ and the no-stream $[F(7,133)=6.592, p<.01]$ sequences. The interaction between target presence and probe position was, however, significant only for the fullstream sequences $[F(7,133)=18.483, p<.001]$. An examination of the simple effect of target presence at each probe position revealed significant processing deficits at the $+1,+2$, +3 , and +5 positions. The interaction between target presence and probe position did not reach significance for no-stream sequences $[F(7,133)=1.942$, $p=.14]$, and the tests of the simple effects showed that there were no significant processing deficits at any position on these trials. Clearly, a significant probe processing deficit, which decreased as the SOA between the target and the probe increased, was apparent only when the sequences included distracting sounds.

We evaluated the three-way interaction between target judgment, target presence, and probe position by examining whether the difference in probe processing accuracy between the target-present and the target-absent conditions was affected by different probe positions for the target-attended and target-ignored sequences separately. This analysis revealed that the main effect of target presence was significant when the participants were instructed to attend to the target $[F(1,19)=39.699, p<.001]$ and when they were instructed to ignore it $[F(1,19)=9.491$, $p<.01]$. The main effect of probe position was also significant when the participants were instructed to attend to the target $[F(7,133)=18.980, p<.001]$ and when they were instructed to ignore it $[F(7,133)=10.686, p<$ $.001]$. Similarly, a significant interaction between target presence and probe position was apparent both when the participants were instructed to attend to the target $[F(7,133)=14.119, p<.001]$ and when they were instructed to ignore it $[F(7,133)=7.547, p<.001]$. Tests of the simple effect of target presence indicated a significant 
probe processing deficit at the $+1,+2$, and +3 positions when the target was attended. In contrast, when the target was ignored, a significant deficit in processing the probe was apparent only at the +1 and +2 positions. A 2 (target judgment required vs. not required) $\times 2$ (target presence) within-subjects ANOVA performed for each probe position revealed significant interactions between target presence and target judgment at the +1 and +3 positions (both $p$ s $<.05)$. These effects arose because the probe processing deficit was larger when the target was attended than when it was ignored at these two positions. Thus, a larger and more enduring probe processing deficit was associated with the requirement to attend to the target.

\section{Probe Detection in Each of the Four Main Conditions}

We also examined performance separately for each principal condition with 2 (target presence) $\times 8$ (Probe Positions +1 through +8 ) within-subjects ANOVAs using as the dependent variable the conditional probability of accurate probe detection when the probe was present given a correct target detection response. In the interest of avoiding redundancy, only $p$ values for significant effects are reported below. Performance in each condition is presented graphically in Figure 1.

No-stream sequences with the target attended. The analysis revealed significant main effects of target presence $(p<.01)$ and probe position $(p<.01)$. Simple effect tests indicated that there were no significant process- ing deficits at any temporal position. Thus, although there was an overall probe processing deficit, it did not reach significance at any probe position.

No-stream sequences with the target ignored. The analysis revealed a significant main effect of probe position only $(p<.05)$. Further analysis confirmed that there was no significant effect of probe processing deficit at any position.

Full-stream sequences with the target attended. The analysis revealed significant main effects of target presence $(p<.001)$ and probe position $(p<.001)$ and a significant interaction between the two $(p<.001)$. Significant probe processing deficits were apparent at the $+1,+2,+3$, and +5 positions. Thus, in this condition the probe processing deficit reached significance, and its magnitude decreased as the time between the target and the probe increased.

Full-stream sequences with the target ignored. Main effects of target presence $(p<.01)$ and probe position $(p<.001)$ were significant, as was the interaction between the two $(p<.001)$. Simple effect tests revealed a significant probe processing deficit at the +1 and +2 positions. Thus, in this condition the probe processing deficit also reached significance, and its magnitude decreased as the time between the target and the probe increased also.

Finally, we determined whether or not there was any difference in the magnitude of the probe processing deficit at any position for the two full-stream conditions. A 2 (target judgment required vs. not required) $\times 2$ (target

\section{No Stream With Target Ignored}

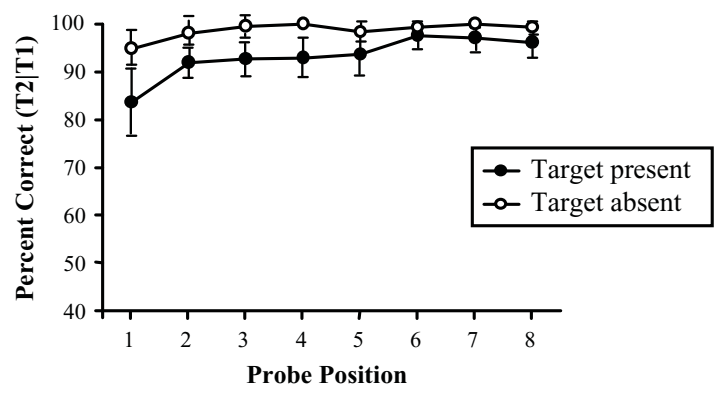

Full Stream With Target Attended

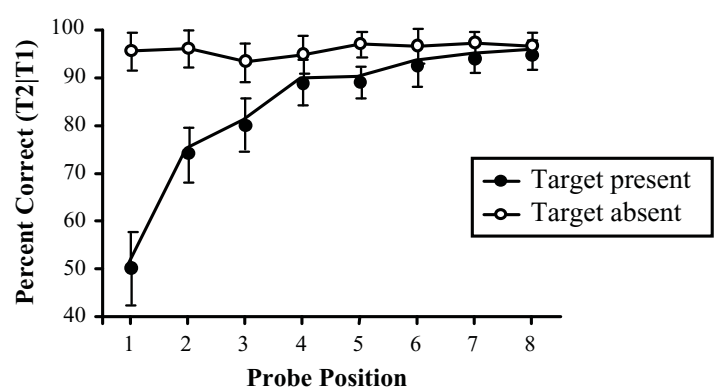

Full Stream With Target Ignored

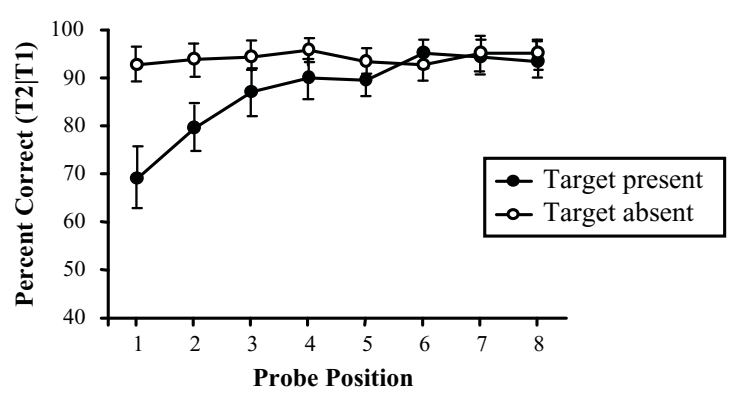

Figure 1. Probe detection accuracy in Experiment 1 as a function of the presence of distractors (no stream vs. full stream) both when the target was to be attended and when it was to be ignored. Error bars represent \pm 1 standard error. 
presence $) \times 8$ (Probe Positions +1 through +8 ) withinsubjects ANOVA revealed a significant three-way interaction between target judgment, target presence, and probe position $[F(7,133)=2.699, p<.05]$. At each probe position, 2 (target judgment required vs. not required) $\times 2$ (target presence) ANOVAs revealed a significant interaction at the +1 position $(p<.01$; all other $p \mathrm{~s}>.10)$. Thus, for the full-stream condition the probe processing deficit was significantly larger at the +1 position when the target was attended than when the target was ignored.

\section{Discussion}

Experiment 1 was conducted to examine whether or not (1) the presence of distractors affected the magnitude of the auditory $\mathrm{AB}$ and (2) a low-level masking account may explain the auditory AB. In general, a main effect of target presence and an interaction between target presence and probe position have been interpreted as indicating the occurrence of an AB (Seiffert \& Di Lollo, 1997). Both of these criteria were met in Experiment 1 for the full-stream sequences but not for the no-stream sequences. It is clear, therefore, that the auditory $\mathrm{AB}$ depends largely on the presence of distractor sounds. This result, obtained for nonverbal material, is consistent with the auditory study of Tremblay et al. (2005), in which spoken syllables were used.

The $A B$ deficit was larger when the target was attended than when it was ignored, and this suggests that it results at least in part because of an attentional limitation rather than because of sensory masking. It is, however, interesting to note that a significant $\mathrm{AB}$ arose for the full-stream sequences even when the target was ignored. One possible explanation for this result is that sensory masking of the probe by the target may have caused the probe processing deficit when the target was ignored. However, if this were the case, then a robust $\mathrm{AB}$ should have been apparent even for the no-stream sequences. This was, of course, not the case. A second possibility, which seems more likely to be correct, is that the target elicited involuntary attentional capture even when the participants were instructed to ignore it. A similar suggestion regarding visual $\mathrm{AB}$ has been made by Jiang and Chun (2001; see also Folk, Leber, \& Egeth, 2002), who have argued that a visual $\mathrm{AB}$ can be produced when a target elicits involuntary attentional capture. However, "compared with the explicit requirement of encoding the identity of T1, involuntary attentional capture exerted less demand on the limited resources responsible for the AB" (Jiang \& Chun, 2001, p. 669). Thus, the $A B$ was larger when the target was attended than when it was ignored.

\section{EXPERIMENT 2}

The results of Experiment 1 showed clearly that the presence of distractors is necessary to produce an auditory AB. In Experiment 2, we addressed the question of whether or not some distractors are more important in this regard than others. Several studies of visual $\mathrm{AB}$ have been conducted to investigate the local effects of the distractors immediately following the target and those immediately following the probe. For example, Raymond et al. (1992) found that the visual $\mathrm{AB}$ was eliminated when the item immediately following the target (i.e., the +1 item) was deleted. Both Chun and Potter (1995) and Seiffert and Di Lollo (1997) found that the visual AB was much reduced when the +1 item was deleted. Raymond et al. (1995) reported that the magnitude of the visual $\mathrm{AB}$ was reduced when the discriminability between the target and the +1 item was increased. Chun and Potter (1995) found that the $\mathrm{AB}$ was reduced when there was high discriminability between the target and the +1 item and between the probe and the distractor immediately following the probe.

In contrast with these studies of the visual AB, Mondor (1998) showed that replacing the +1 item with silence did not reduce the magnitude of the auditory AB. In Experiment 2 , we sought to replicate this theoretically important result and to examine the effect of deleting the item immediately preceding the target or immediately preceding or following the probe, to determine whether or not elimination of any of these specific distractors has a particularly pronounced influence on the magnitude of the auditory $\mathrm{AB}$.

According to the attentional gate model (Raymond et al., 1992), the presence of the +1 item initiates an inhibition mechanism to prevent interference with target processing, and it is this inhibition that causes the $\mathrm{AB}$. Thus, deleting the +1 item should eliminate or at least reduce the magnitude of the auditory $\mathrm{AB}$. The two-stage model (Chun \& Potter, 1995; Giesbrecht \& Di Lollo, 1998; Jolicœur, 1998) is based on the idea that processing of the probe must wait until target processing is complete. During this delay, the memorial representation of the probe may deteriorate either because of decay or because of overwriting generated by postprobe distractors. The probe processing delay may be extended if the +1 item is processed along with the target, since this increases the difficulty of target processing. This is particularly the case during short-term consolidation, which refers to "the process of encoding information into short-term memory" (Jolicour, 1998, p. 1028) and relies on a capacity-limited cognitive processing resource. Thus, the magnitude of the $\mathrm{AB}$ increases as the probe processing delay increases. According to this model, eliminating the +1 item can reduce target processing difficulty, thereby reducing the magnitude of the visual AB. Finally, the competition hypothesis (Shapiro et al., 1994) proposes that the target, the probe, and the distractors immediately following each of them gain access to VSTM and compete for limited resources. Thus, deleting the +1 item should reduce competition and thereby improve probe detection. In short, all three theories predict a reduction in the magnitude of $\mathrm{AB}$ when the +1 item is replaced by silence.

By contrast, these three approaches offer different predictions regarding deletions of distractors following the probe. Whereas the attentional gate model (Raymond et al., 1992) is silent with respect to the influence of the 
postprobe distractor, the two-stage model (Chun \& Potter, 1995; Giesbrecht \& Di Lollo, 1998; Jolicœur, 1998) suggests that this distractor has an important effect on the magnitude of the $\mathrm{AB}$ because it may overwrite or substitute for the probe. Thus, replacing this item with silence should reduce the magnitude of the AB. According to the competition hypothesis (Shapiro et al., 1994), the item following the probe also could gain access to VSTM because of its close temporal proximity to the probe, and this would result in competition for cognitive resources. Therefore, deleting the item following the probe should reduce this competition as well as the magnitude of the AB.

None of the three theories places any special emphasis on the distractor item immediately preceding the target or the probe.

\section{Method}

\section{Participants}

Twenty undergraduate students enrolled in an introductory psychology course at the University of Manitoba participated in the experiment in exchange for course credit. All of the participants reported normal hearing.

\begin{abstract}
Materials
The computer and sound system and the sounds used were identical to those used in Experiment 1.

\section{Procedure}

On each trial, the participants were presented with a sequence of sounds that was similar to the full-stream sequences in Experiment 1 except that one distractor sound was replaced by a silent period of the same duration. This silent period could occur immediately prior to or immediately following either the target or the probe. When a silent period was presented prior to the target or following the probe, the probe could occur in one of the first eight temporal positions following the target. For each of these two conditions, the participants completed 32 practice trials and 256 experimental trials. In contrast, when the silent period followed the target or preceded the probe, the probe was presented in one of Temporal Positions +2 through +8 . For each of these two conditions, the participants completed 32 practice trials and 224 experimental trials. Each participant was required to complete all four blocks. A Latin square was used to balance the order of the blocks. In the practice trials, each participant was required to meet a criterion of $60 \%$ correct on the probe judgments before beginning the experimental trials. Eighteen participants met this criterion after a single block of practice trials, whereas 2 participants required two blocks of trials to reach this level of performance. The participants were required to listen for both the target and the probe on all trials. Because the results of Experiment 1 showed that low-level masking was not a principle cause of the auditory $A B$, we did not include a condition in which listeners were instructed to ignore the target. As in Experiment 1, the target and the probe were each presented on $50 \%$ of the trials. All other methodological details were the same as in Experiment 1.
\end{abstract}

\section{Results}

\section{Target Detection}

The probability of a correct response to the target task across all conditions was $.942(S E=.013)$. The probability of a correct response on a target-present trial (hit) was .953 , and the probability of an incorrect response on a target-absent trial (false alarm) was .069. Thus, average $d^{\prime}$ was 3.14. In summary, then, as in Experiment 1, the participants found the target-detection task quite easy.

A 4 (silence position: prior to vs. following the target vs. the probe) $\times 2$ (probe presence) within-subjects ANOVA was conducted using as the dependent variable the probability of accurate target detection when the target was present. The analysis revealed a marginally significant main effect of probe presence $[F(1,19)=3.516, p=.08]$, which arose because there was a slight increase in the accuracy of target detection when the probe was present. None of the other effects reached significance (all $p \mathrm{~s}>.10$ ).

A 4 (silence position prior to vs. following the target vs. the probe) $\times 7$ (Probe Positions +2 through +8 ) withinsubjects ANOVA was conducted using as the dependent variable the probability of accurate target detection when both the target and the probe were presented. Performance was examined only for Positions +2 through +8 because these positions were common to all conditions. The results revealed only a marginally significant main effect of probe position $[F(6,114)=2.358, p=.07]$ owing to a slight increase in the accuracy of target detection as probe position increased. None of the other effects approached significance (all other $p \mathrm{~s}>.30$ ).

Finally, to examine whether or not target processing difficulty was reduced after deletion of one item, we compared target detection performance in each condition of the present experiment with performance apparent in the corresponding conditions of Experiment 1 in which the participants were to listen for both the target and the probe presented in full-stream sequences. A one-way between-subjects ANOVA using percent correct as the dependent measure revealed no significant effects $(p>.30)$, whereas an analysis of $d^{\prime}$ revealed a marginally significant improvement in performance when the item following the target was replaced by silence $[F(1,38)=3.602, p=.07$; all other $p \mathrm{~s}>.30]$.

\section{Probe Detection}

Overall probe detection accuracy on trials on which a correct response was made for the target judgment was $.893(S E=.018)$. The probability of a correct response on a probe-present trial (hit) was .890 , and the probability of an incorrect response on a probe-absent trial (false alarm) was .103. Thus, average $d^{\prime}$ was 2.48 .

A 4 (silence position prior to vs. following the target vs. the probe) $\times 2$ (target presence) $\times 7$ (Probe Positions +2 through +8 ) within-subjects ANOVA was conducted using as the dependent variable the conditional probability of accurate probe detection when the probe was present given a correct target detection response. Performance was examined only for Positions +2 through +8 because these positions were common to all conditions. This analysis revealed significant main effects of target presence $[F(1,19)=35.359, p<.001]$, silence position $[F(3,57)=$ $4.193, p<.05]$, and probe position $[F(6,114)=17.440$, $p<.001]$. In general, the participants detected the probe 
more accurately when the target was absent, when long SOAs were present between the target and the probe, and when a silent period was present following the probe.

Significant two-way interactions between target presence and silence position $[F(3,57)=4.504, p<.05]$, target presence and probe position $[F(6,114)=20.623$, $p<.001]$, and silence position and probe position $[F(18,342)=2.486, p<.01]$ were also apparent. However, the three-way interaction between target presence, silence position, and probe position was not significant $(F<1)$. More detailed analysis of the significant interactions is included in the next section.

\section{Probe Detection in Each of the Four Main Conditions}

We used target presence $\times$ probe position withinsubjects ANOVAs to examine performance separately for each of the principal conditions defined by the placement of the silent period. We used as the dependent variable the conditional probability of accurate probe detection when the probe was present given a correct target detection response. In the silent period prior to the target and following the probe condition, the analyses were based on $8(+1$ through +8$)$ probe positions. By contrast, in the silent period following the target and prior to the probe condition, the analysis was based on $7(+2$ through +8$)$ probe positions. In addition, performance in each condition was compared with that obtained for the full-stream sequences when the target was attended in Experiment 1. For the silent period prior to the target and following the probe condition, 2 (Experiment 1 vs. Experiment 2, between subjects) $\times 2$ (target presence) $\times 8$ (Probe Positions +1 though +8 ) split-plot ANOVAs were conducted using as the dependent variable the conditional probability of accurate probe detection when the probe was present given a correct target detection response. For the silent period following the target and prior to the probe conditions, $2 \times 2 \times 7$ (Probe Positions +2 though +8 ) splitplot ANOVAs were conducted. Four effects (i.e., a main effect of experiment, an interaction between experiment and target presence, an interaction between experiment and probe position, and an interaction between experiment, target presence, and probe position) were used as indicators of difference. In the interest of avoiding redundancy, only $p$ values for significant effects are reported below. Performance in each condition is presented graphically in Figure 2.

Silent period prior to the target. The two-way ANOVA revealed significant main effects of target presence $(p<.001)$ and probe position $(p<.001)$ as well as a significant interaction between these two factors $(p<$ $.001]$. Analysis of the simple effects revealed significant probe processing deficits at the $+1,+2,+3$, and +4 positions. The three-way ANOVA revealed that none of the four effects was significant (all $F_{\mathrm{s}}<1$ ). Thus, performance in this condition did not differ significantly from that in the full-stream sequences used in Experiment 1. It appears that the magnitude of the auditory $\mathrm{AB}$ is not significantly influenced by elimination of the distractor preceding the target.

\section{Silence Prior to the Target}

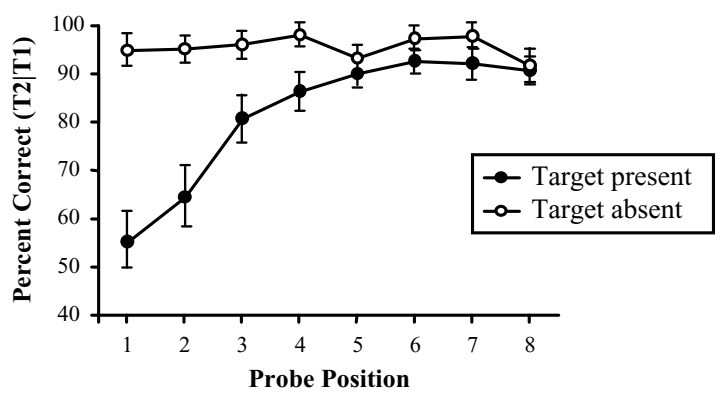

Silence Prior to the Probe

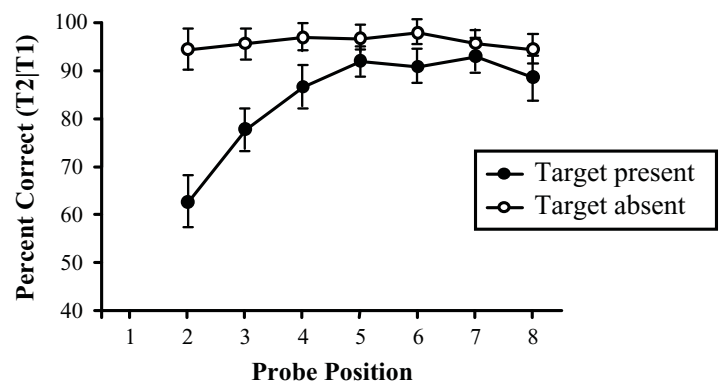

\section{Silence Following the Target}

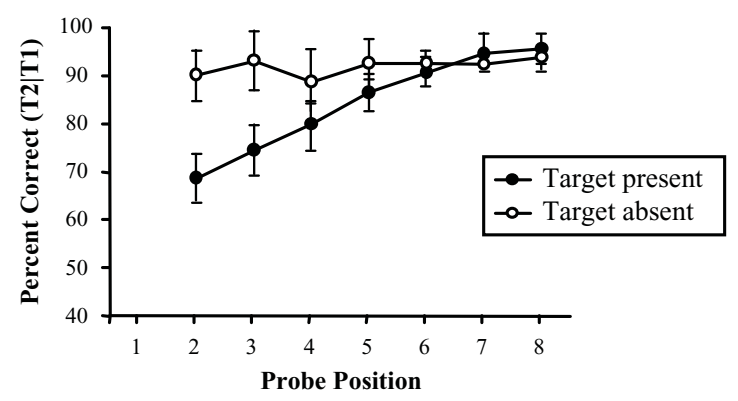

Silence Following the Probe

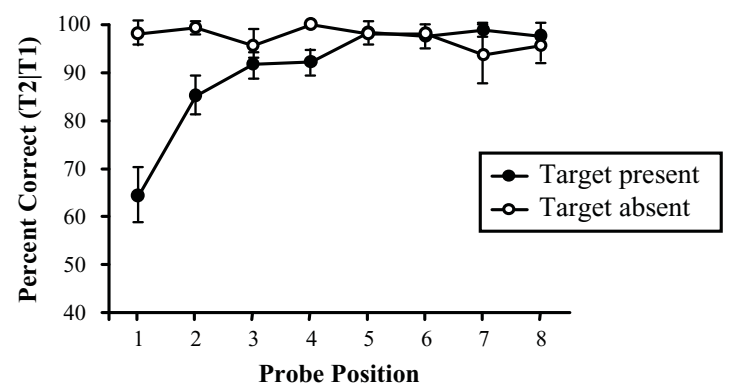

Figure 2. Probe detection accuracy in Experiment 2 when a silent period was inserted prior to or following either the target or the probe. Error bars represent \pm 1 standard error. 
Silent period following the target. The two-way ANOVA revealed significant main effects of target presence $(p<.05)$ and probe position $(p<.001)$ and a significant interaction between the two $(p<.001)$. Analysis of the simple effects revealed significant probe processing deficits at the +2 and +3 positions. The three-way ANOVA revealed that none of the four effects was significant (all $p \mathrm{~s}>.30$ ). Thus, performance in this condition also did not differ significantly from that in the full-stream sequences used in Experiment 1 in which the target was attended. It appears that the magnitude of the auditory $\mathrm{AB}$ is not significantly influenced by elimination of the distractor following the target.

Silent period prior to the probe. The two-way ANOVA revealed significant main effects of target presence $(p<.001)$ and probe position $(p<.001)$ and a significant interaction between the two $(p<.001)$. Further analysis indicated significant probe processing deficits at the +2 and +3 positions. The three-way ANOVA revealed that none of the four effects was significant (all $p s>.20$ ). Thus, performance in this condition did not differ significantly from that in the full-stream sequences used in Experiment 1. It appears that the auditory AB is not significantly influenced by elimination of the distractor prior to the probe.

Silent period following the probe. The two-way ANOVA revealed significant main effects of target presence $(p<.05)$ and probe position $(p<.001)$ and a significant interaction between the two $(p<.001)$. Significant probe processing deficits were apparent at the $+1,+2$, and +4 positions. The three-way ANOVA revealed a marginally significant main effect of experiment $[F(1,38)=$ $3.146, p=.08]$ and a significant interaction between experiment and target presence $[F(1,38)=6.415, p<$ $.05 ; p>.30$ for the other two effects). Furthermore, a 2 (Experiment 1 vs. Experiment 2, between-subjects] $\times 2$ (target presence) split-plot ANOVA at each probe position revealed there was an interaction at the +1 position $(p<$ .05 ; all other $p \mathrm{~s}>.10$ ). These results establish that the magnitude of the auditory $\mathrm{AB}$ is reduced when the distractor following the probe is replaced by a silent period.

\section{Discussion}

This experiment was conducted to determine whether or not the placement of a silent period at a specific temporal position within a sequence of sounds has any influence on the magnitude of the auditory AB. Replacing the item preceding the target or preceding the probe was found to have no substantive effect on the magnitude of the $\mathrm{AB}$. If either of these items had interfered with target and probe processing, then deleting it should have reduced the magnitude of the AB. However, it appears that neither target processing nor probe processing is influenced significantly by preceding distractors.

Similarly, and in direct contrast with the results reported in studies of the visual $\mathrm{AB}$, replacing the item following the target with a silent period had no significant effect on the magnitude of the auditory AB (see Mondor, 1998, for a similar result). As we discussed above, such a result is inconsistent with the attentional gate model, the competition hypothesis, and the two-stage model because all of these require that deletion of the +1 item improve probe detection, albeit for quite different reasons.

We did obtain solid evidence that the magnitude of the $\mathrm{AB}$ was reduced when a silent period followed the probe. This result is consistent with the two-stage theory (Chun \& Potter, 1995; Giesbrecht \& Di Lollo, 1998; Jolicœur, 1998 ) and the competition hypothesis (Shapiro et al., 1994) but not with the attentional gate model (Raymond et al., 1992). According to the two-stage model, replacing the item following the probe with silence should reduce the possibility of the probe's being overwritten, thereby directly reducing the magnitude of the auditory $\mathrm{AB}$. According to the competition hypothesis, deleting this item will reduce the competition between the target and the probe and should reduce the magnitude of the auditory AB. This result is inconsistent with the attentional gate model because it does not recognize any substantive contribution of the distractor following the probe to the generation of the $\mathrm{AB}$.

It is possible that a modified version of the two-stage model may be able to explain our results because the two-stage model is based on the notion that the item immediately following the target and the item immediately following the probe have different effects (i.e., the +1 item can be processed together with the target, thereby increasing the difficulty of target processing during shortterm consolidation and increasing the magnitude of the $\mathrm{AB}$, whereas the item immediately following the probe can overwrite or substitute the probe, thereby causing a larger $\mathrm{AB}$ deficit). It seems to us quite likely that there is no substantive effect of inserting a silent period following the target because the +1 item is not processed along with the target, and so it does not affect its short-term consolidation. Thus, although deleting the +1 item did reduce the difficulty of target processing, this may well be because of a reduction in the backward masking of the target, which influences the sensory registration of the target but not its short-term consolidation. This view is consistent with the findings of visual studies that the short-term consolidation of the target, rather than its initial sensory registration, influences the magnitude of the AB (Jolicour, 1998; Olson, Chun, \& Anderson, 2001). In keeping with the two-stage model, it does appear that in audition the distractor immediately following the probe can cause overwriting of the probe and thus produce a larger $\mathrm{AB}$ deficit.

\section{EXPERIMENT 3}

In Experiment 2, deleting the +1 item did not affect the magnitude of the auditory $\mathrm{AB}$. This result is quite different from that apparent in studies of the visual $\mathrm{AB}$, which have shown that this item plays an important role in producing the probe processing deficit. Although it may be that in audition the +1 item is not processed together with the target, another explanation is possible. This has to do with 
the duration of the silent period following the target that was created when the +1 item was replaced with silence. In studies of the visual $\mathrm{AB}$ in which a large reduction in the magnitude of the $\mathrm{AB}$ was apparent when the +1 item was deleted, SOA between the target and the subsequent distractor (presented in the +2 position) ranged from $180 \mathrm{msec}$ (Raymond et al., 1992; Seiffert \& Di Lollo, 1997) to $200 \mathrm{msec}$ (Chun \& Potter, 1995), with the total duration of the silent period ranging from $100 \mathrm{msec}$ (Chun \& Potter, 1995) to $165 \mathrm{msec}$ (Raymond et al., 1992; Seiffert \& Di Lollo, 1997). In contrast, in Experiment 2, when the +1 item was replaced with silence, SOA between the target and the +2 distractor was equal to $160 \mathrm{msec}$ and the ISI was $130 \mathrm{msec}$. It is possible that the lack of any effect of the +1 item that we observed may have occurred either because of the shorter SOA between the target and the +2 item or because of the brief silent period relative to that used in some of the previous visual studies.

In the present experiment, we again examined the influence of the distractor sound following the target under conditions in which the SOA between successive sounds was increased to $95 \mathrm{msec}$. (Each item was still presented for $30 \mathrm{msec}$, but the ISI was increased to $65 \mathrm{msec}$.) Thus, when the +1 item was replaced by silence, the resulting SOA between the target and the +2 item was 190 msec and ISI was $160 \mathrm{msec}$. Because in the Experiment 2 deletion of the item immediately prior to either the target or the probe had no effect on the auditory $A B$ and, to our knowledge, no visual study has shown either of these distractors to have an effect on the visual $A B$, we did not specifically study their effects in the present experiment. In different conditions, we again examined the effect of replacing with silence the item following the target or following the probe. We also examined performance in a baseline condition in which listeners were presented with an uninterrupted stream of sounds. A within-subjects design was used to eliminate any possibility that a difference in the pattern of performance obtained in the three conditions could be attributed to idiosyncratic differences between groups of participants.

Method
Participants
Twenty-four undergraduate students enrolled in an introductory
psychology course at the University of Manitoba participated in the
experiment in exchange for course credit. All the participants re-
ported normal hearing.

\section{Materials}

The computer and sound system and the sounds used were identical to those used in Experiments 1 and 2.

\section{Procedure}

Performance was examined in three separate conditions. In the baseline condition, the listeners were presented with a complete sequence of 16 sounds on each trial. These sequences were the same as those used in the full-stream condition of Experiment 1, except that the ISI between successive items was increased from 50 to $65 \mathrm{msec}$ and the probe could occur only in one of the first seven temporal positions following the target. The SOA between the target and the +8 item in the previous experiment was $640 \mathrm{msec}$. Because we wished to maintain a similar SOA in the present experiment, we examined performance for only the first seven temporal positions. Thus, the SOA between the target and the +7 item was $665 \mathrm{msec}$. In the second condition, the sound immediately following the target was replaced by silence and the probe was presented in one of Positions +2 through +7 . Finally, in the third condition, the sound following the probe was replaced with a silent period and the probe was presented in one of Positions +1 through +7 . As in Experiments 1 and 2, the target and the probe were each presented on $50 \%$ of the trials. The participants were required to listen for both the target and the probe on all trials.

The participants completed 32 practice trials for each of the three conditions. Each participant was required to meet a criterion of $60 \%$ correct on the probe judgments before beginning the experimental trials. Twenty-one participants met this criterion after a single block of practice trials, whereas 3 participants required two blocks of trials to reach this level of performance. All the participants completed 224 experimental trials in the full-stream condition and in the condition in which the sound immediately following the probe was replaced by silence. They also completed 192 experimental trials in the condition in which the item immediately following the target was deleted. The order in which these three conditions were performed was completely counterbalanced across participants. All other methodological details were same as in Experiments 1 and 2.

\section{Results}

\section{Target Detection}

The probability of a correct response to the target task across all conditions was $951(S E=.007)$. The probability of a correct response on a target-present trial (hit) was .942 , and the probability of an incorrect response on a target-absent trial (false alarm) was .040. Thus, average $d^{\prime}$ was 3.32 .

A 3 (stream condition: full stream vs. silence following the target vs. silence following the probe) $\times 2$ (probe presence) within-subjects ANOVA was conducted using as the dependent variable the probability of accurate target detection when the target was present. The analysis revealed a significant main effect of stream condition $[F(2,46)=$ 3.917, $p<.05]$. Multiple comparisons using the Bonferroni adjustment revealed that the accuracy of target detection increased only when the +1 item was deleted $[F(1,23)=9.971, p<.01]$. To further examine whether or not target processing difficulty was influenced by deletion of an item, multiple planned comparisons using the Bonferroni adjustment were conducted on $d^{\prime}$ for target detection. The results also revealed an increase in $d^{\prime}$ only when the +1 item was replaced by silence $[F(1,23)=$ $6.337, p<.025]$. In summary, both the accuracy and the $d^{\prime}$ results indicated that the difficulty of target processing was reduced when the +1 item was replaced by silence. Neither the main effect of probe presence nor the interaction between stream condition and probe presence reached significance (both $p \mathrm{~s}>.30$ ). Thus, the presence of the probe did not affect the accuracy of target detection.

A 3 (stream condition: full vs. silence following the target vs. silence following the probe) $\times 6$ (Probe Positions +2 through +7 ) within-subjects ANOVA was conducted using as the dependent variable the probability of accurate target detection when both the target and the probe were 
presented. The results did not reveal any significant effects (all $F \mathbf{s}<1$ ). Thus, the accuracy of target detection did not vary as a function of probe position.

\section{Probe Detection}

Overall probe detection accuracy on trials on which a correct response was made for the target judgment was $.908(S E=.011)$. The probability of a correct response on a probe-present trial (hit) was .899 , and the probability of an incorrect response on a probe-absent trial (false alarm) was .084. Thus, average $d^{\prime}$ was 2.66 .

A 3 (stream condition: full vs. silence following the target vs. silence following the probe) $\times 2$ (target presence) $\times 6$ (Probe Positions +2 through +7 ) withinsubjects ANOVA was conducted using as the dependent variable the conditional probability of accurate probe detection when the probe was present given a correct target detection response. Performance was examined only for Positions +2 through +7 because these positions were common to all conditions. This analysis revealed significant main effects of target presence $[F(1,23)=17.431$, $p<.001]$ and probe position $[F(5,115)=4.475, p<.05]$. However, the main effect of stream was not significant $[F(2,46)=1.714, p=.20]$. In general, the participants detected the probe more accurately when the target was absent and when the probe occurred at long SOAs.

Significant two-way interactions between stream and target presence $[F(2,46)=4.128, p<.05]$ and between target presence and probe position $[F(5,115)=5.904$, $p<.01]$ were apparent. However, neither the interaction between stream and probe position $(F<1)$ nor the threeway interaction between stream, target presence, and probe position $(F<1)$ was significant.

\section{Probe Detection in Each of the Three Main Conditions}

We examined performance separately for each of the three principal conditions defined by the placement of the silent period using two-way within-subjects ANOVAs (target presence $\times$ probe position) of the conditional probability of accurate probe detection when the probe was present given a correct target detection response as the dependent variable. For full stream and silent period following the probe sequences, the analyses were based on seven probe positions $(+1$ through +7$)$. In contrast, for the silent period following the target sequences, the analysis was based on six probe positions $(+2$ through +7 ). Performance in each condition is presented graphically in Figure 3.

Full stream. The two-way ANOVA revealed significant main effects of target presence $(p<.001)$ and probe position $(p<.001)$ as well as a significant interaction between these two factors $(p<.001)$. Analysis of the simple effects revealed significant probe processing deficits at the $+1,+2$, and +3 positions. This auditory $\mathrm{AB}$ is quite similar to that observed in Experiment 1 under the same condition.

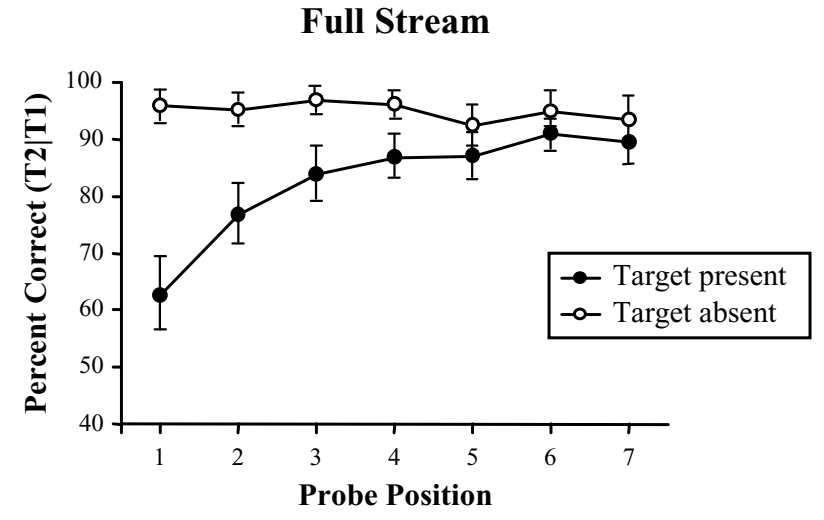

Silence Following the Target

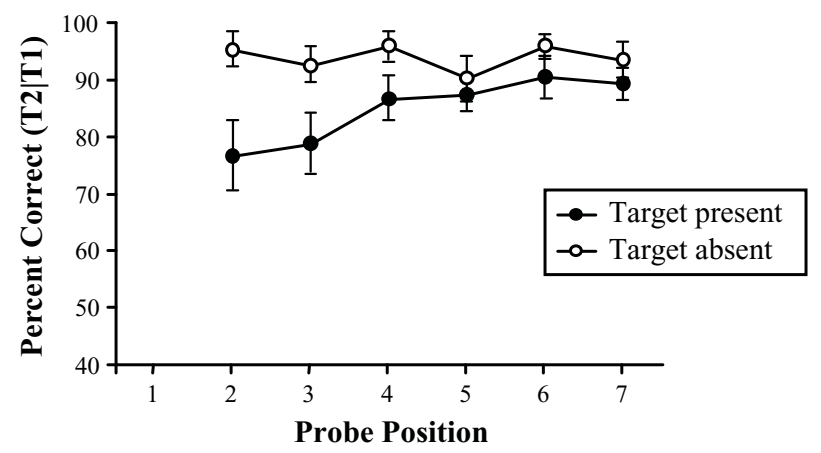

Silence Following the Probe

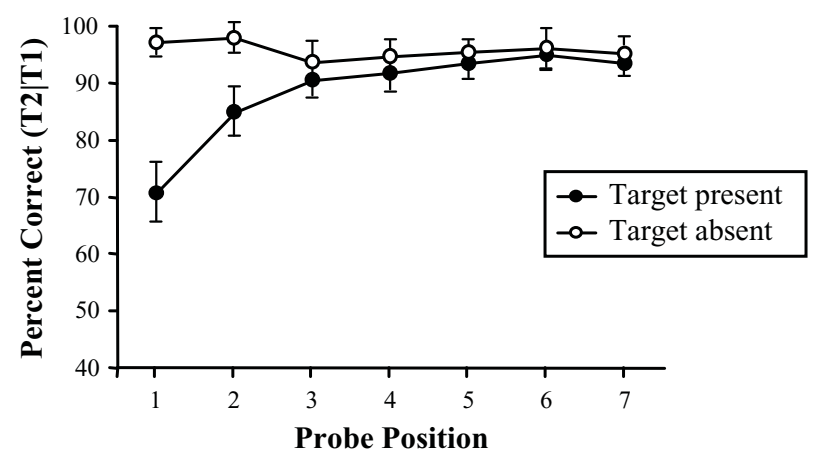

Figure 3. Probe detection accuracy in Experiment 3 in the full stream, silent period following the target, and silent period following the probe conditions. Error bars represent \pm 1 standard error.

Silent period following the target. The two-way ANOVA revealed significant main effects of target presence $(p<.01)$ and probe position $(p<.05)$ and a significant interaction between the two $(p<.05)$. Analysis of the simple effects revealed significant probe processing deficits at the +2 and +3 positions. The magnitude of the $\mathrm{AB}$ apparent in this condition was compared with that observed in the full-stream condition using a 2 (stream: full vs. blank following the target) $\times 2$ (target presence) $\times 6$ Probe Positions +2 through +7 ) within-subjects ANOVA. 
Only performance for Positions +2 through +7 was examined because these positions were common to all conditions. The three-way ANOVA revealed that none of the four effects (i.e., the main effect of stream and the interactions between [1] stream and target presence, [2] stream and probe position, and [3] stream, target presence, and probe position) was significant (all $F \mathrm{~s}<1$ ). Thus, the analysis indicated no difference in performance in the two conditions.

Silent period following the probe. The analysis revealed significant main effects of target presence $(p<$ $.001)$ and probe position $(p<.01)$ and a significant interaction between the two $(p<.001)$. Analysis of the simple effects revealed significant probe processing deficits at the +1 and +2 positions. A comparison of performance in this condition with that in the full-stream condition using a 2 (stream: full vs. blank following the target) $\times 2$ (target presence) $\times 7$ (Probe Positions +1 through +7 ) withinsubjects ANOVA revealed only a significant interaction between stream and target presence $[F(1,23)=6.344, p<$ .05 ; all other $p \mathrm{~s}>.10$ ). At each position, 2 (stream) $\times 2$ (target presence) within-subjects ANOVAs revealed that there was an interaction at the +3 position $(p<.05$; all other $p \mathrm{~s}>.10)$. These results indicated that the magnitude of the auditory $\mathrm{AB}$ was reduced when the distractor following the probe was replaced by a silent period.

\section{Discussion}

Experiment 3 was conducted to determine whether or not the influence of the posttarget and postprobe distractors may depend on the SOA and the within-subjects design. Thus, relative to Experiment 2, both SOA and the duration of the silent period following either the target or the probe was lengthened using a within-subjects design. These changes had only a very minor influence on performance. Thus, as was the case in Experiment 2, the magnitude of the $A B$ was reduced significantly only when a silent period followed the probe; replacing the item following the target with a silent period had no significant effect on the magnitude of the auditory AB. In addition, with regard to the difficulty of target processing, in Experiment $2 d^{\prime}$ was increased after the +1 item was deleted, and in the present experiment both $d^{\prime}$ and accuracy increased. Thus, lengthening the silent period following the target did reduce the difficulty of target processing. However, this did not result in a reduction in the magnitude of the auditory $\mathrm{AB}$. These results, then, support the modified version of the two-stage model that we suggested in Experiment 2 .

\section{EXPERIMENT 4}

The results of Experiment 1 showed that the presence of distractor sounds is critical to the production of an auditory $\mathrm{AB}$. The results of Experiments 2 and 3 suggest that it is the distractor immediately following the probe that is of most importance in this regard. Interestingly, however, a robust probe processing deficit remained even when the item immediately following the probe was replaced by silence. This raises the possibility that the other distractors following the probe may also contribute to the generation of the auditory AB. Indeed, studies of the visual AB (Giesbrecht \& Di Lollo, 1998; Kawahara, Di Lollo, \& Enns, 2001; Kawahara et al., 2003) have shown that the postprobe distractor items are important in producing the AB. In Experiment 4, we addressed this possibility by replacing all of the items following the probe with a silent period of equivalent duration.

Method
Participants
Twenty undergraduate students enrolled in an introductory psy-

Twenty underge course at the University of Manitoba participated in the experiment in exchange for course credit. All the participants reported normal hearing.

\section{Materials}

The computer and sound system and the sounds used were identical to those used in Experiments 1, 2, and 3.

\section{Procedure}

The conditions of this experiment were similar to those of Experiments 2 and 3 in which the item immediately following the probe was replaced by silence. In contrast with these experiments, however, in Experiment 4 all of the distractors following the probe were replaced by silent periods. In different conditions, SOA between successive sounds was set to either $80 \mathrm{msec}$ (as in Experiment 2) or $95 \mathrm{msec}$ (as in Experiment 3). All the participants completed both conditions, with half of them performing the 80 -msec SOA condition first and the other half completing the $95-\mathrm{msec}$ SOA condition first. Probes were presented in Positions +1 through +8 for the 80 msec SOA sequences (as in Experiment 2), but only in Positions +1 through +7 for the 95-msec SOA sequences (as in Experiment 3).

The participants completed 32 practice trials and 256 experimental trials for $80-\mathrm{msec}$ sequences, and they completed 32 practice trials and 224 experimental trials for 95 -msec sequences. Each participant was required to meet a criterion of $60 \%$ correct on the probe judgments in the practice trials before beginning the experimental trials. All 20 participants passed this criterion after a single block of practice trials. All other methodological details were the same as for the condition in Experiments 2 and 3 in which the sound following the probe was replaced by silence.

\section{Results}

\section{Target Detection}

The probability of a correct response to the target task across all conditions was $.965(S E=.005)$. The probability of a correct response on a target-present trial (hit) was .970 , and the probability of an incorrect response on a target-absent trial (false alarm) was .042. Thus, average $d^{\prime}$ was 3.61. As in Experiment 1,2, and 3, the listeners found the target detection task quite easy.

A 2 (SOA: 80 vs. $95 \mathrm{msec}) \times 2$ (probe presence) withinsubjects ANOVA was conducted using as the dependent variable the probability of accurate target detection when the target was present. The analysis revealed a marginally significant main effect of probe presence $[F(1,19)=$ 3.303, $p=.085$ ] due to the fact that there was a slight 
increase in the accuracy of the target when the probe was present. None of the other effects reached significance (all other $p \mathrm{~s}>.10)$.

A 2 (SOA: 80 vs. $95 \mathrm{msec}$ ) $\times 7$ (Probe Positions +1 through +7) within-subjects ANOVA was conducted using as the dependent variable the probability of accurate target detection when both the target and the probe were presented. The results revealed a significant main effect of probe position $[F(6,114)=3.802, p<.05]$. Thus, the accuracy of target detection did improve as the temporal separation between the target and the probe increased. No other effects were significant (all other $F \mathrm{~s}<1$ ).

\section{Probe Detection}

Overall probe detection accuracy on trials on which a correct response was made for the probe judgment was $.971(S E=.006)$. The probability of a correct response on a probe-present trial (hit) was .970 , and the probability of an incorrect response on a probe-absent trial (false alarm) was .030. Thus, average $d^{\prime}$ was 3.74 .

A 2 (SOA: 80 vs. $95 \mathrm{msec}) \times 2$ (target presence) $\times 7$ (Probe Positions +1 through +7 ) within-subjects ANOVA was conducted using as the dependent variable the conditional probability of accurate probe detection when the probe was present given a correct target detection response. Performance was examined only for Positions +1 through +7 because these positions were common to the two conditions. This analysis revealed significant main effects of SOA $[F(1,19)=4.779, p<.05]$, target presence $[F(1,19)=17.078, p<.01]$, and probe position $[F(6,114)=4.21, p<.05]$. In general, the participants detected the probe more accurately when the SOA of sequence was longer (lower rate of sequence), when the target was absent, and when the SOAs between the target and the probe were longer (i.e., when the probe occurred at long SOAs). A significant two-way interaction between target presence and probe position was apparent $[F(6,114)=$ $7.109, p<.001]$. This interaction arose because of larger probe processing deficits due to a more pronounced effect of target presence at shorter SOAs between the target and the probe than at longer SOAs. However, no other interaction was significant (all other $p \mathrm{~s}>.20$ ).

\section{Probe Detection in Each of the Two Main Conditions}

We examined performance separately for each of the two principal conditions defined by the placement of the silent period using two-way within-subjects ANOVAs (target presence $\times$ probe position) using as the dependent variable the conditional probability of accurate probe detection when the probe was present given a correct target detection response. In addition, performance for the 80-msec SOA sequences was compared to that apparent in Experiment 2, in which sequences with the same SOA were used but only the first distractor sound following the probe was deleted. Similarly, performance for the 95msec sequences was compared to that apparent in Experiment 3 , in which sequences with the same SOA were used but only the first distractor sound following the probe was deleted. For these comparisons, we used split-plot ANOVAs (experiment $\times$ target presence $\times$ probe position) based on the conditional probability of accurate probe detection when the probe was present given a correct target detection response as the dependent variable. All analyses were based on $8(+1$ through +8$)$ and $7(+1$ through +7$)$ probe positions for the 80- and 95-msec SOA sequences, respectively. In the interest of avoiding redundancy, only $p$ values for significant effects are reported below. Performance in each condition is presented graphically in Figure 4.

80-msec SOA sequences. The two-way ANOVA revealed significant main effects of target presence $(p<$ $.01)$ and probe position $(p<.05)$. A significant interaction between these two factors was apparent as well $(p<$ $.01)$. Tests of the effect of target presence at each position revealed a significant probe processing deficit only at the +1 position. The three-way ANOVA indicated a significant interaction between experiment and probe position $(p<.05)$ and between experiment, target presence, and probe position $(p<.01)$. The interaction between experiment and target presence was marginally significant $(p=$ $.08)$. The main effect of experiment was not significant $(p>.20)$. An evaluation of the interaction between experiment and target presence at each probe position revealed a significant effect only at the +1 position $(p<.05)$. Thus, relative to replacing the item immediately following the probe by a silent period, replacing all of the items following the probe by an extended silent period resulted in a reduced probe processing deficit. It is clear, therefore, that the magnitude of the auditory AB is influenced by postprobe distractors other than the one immediately following the probe.

95-msec SOA sequences. The two-way ANOVA revealed a significant main effect of target presence $(p<$ .001 ), a marginally significant main effect of probe position $(p=.06)$, and a marginally significant interaction between these two factors $(p=.10)$. Tests of the effect of target presence at each position did not reveal a significant probe processing deficit at any position. The three-way ANOVA revealed a significant main effect of experiment $(p<.05)$ and significant interactions between experiment and target presence $(p<.05)$ and between experiment, target presence, and probe position $(p<.05)$. The interaction between experiment and probe position was not significant $(p>.20)$. An evaluation of the interaction between experiment and target presence at each probe position revealed a significant effect at the +1 and +2 positions (both $p \mathrm{~s}<.05$ ). Thus, relative to replacing the item immediately following the probe by a silent period, replacing all of the items following the probe by an extended silent period resulted in a greatly reduced probe processing deficit. It is clear that the postprobe distractors also play an important role in generating the auditory $\mathrm{AB}$ in this situation.

\section{Discussion}

This experiment was conducted to examine whether or not the auditory $\mathrm{AB}$ might be fully eliminated when all of the items following the probe are replaced by silence. 

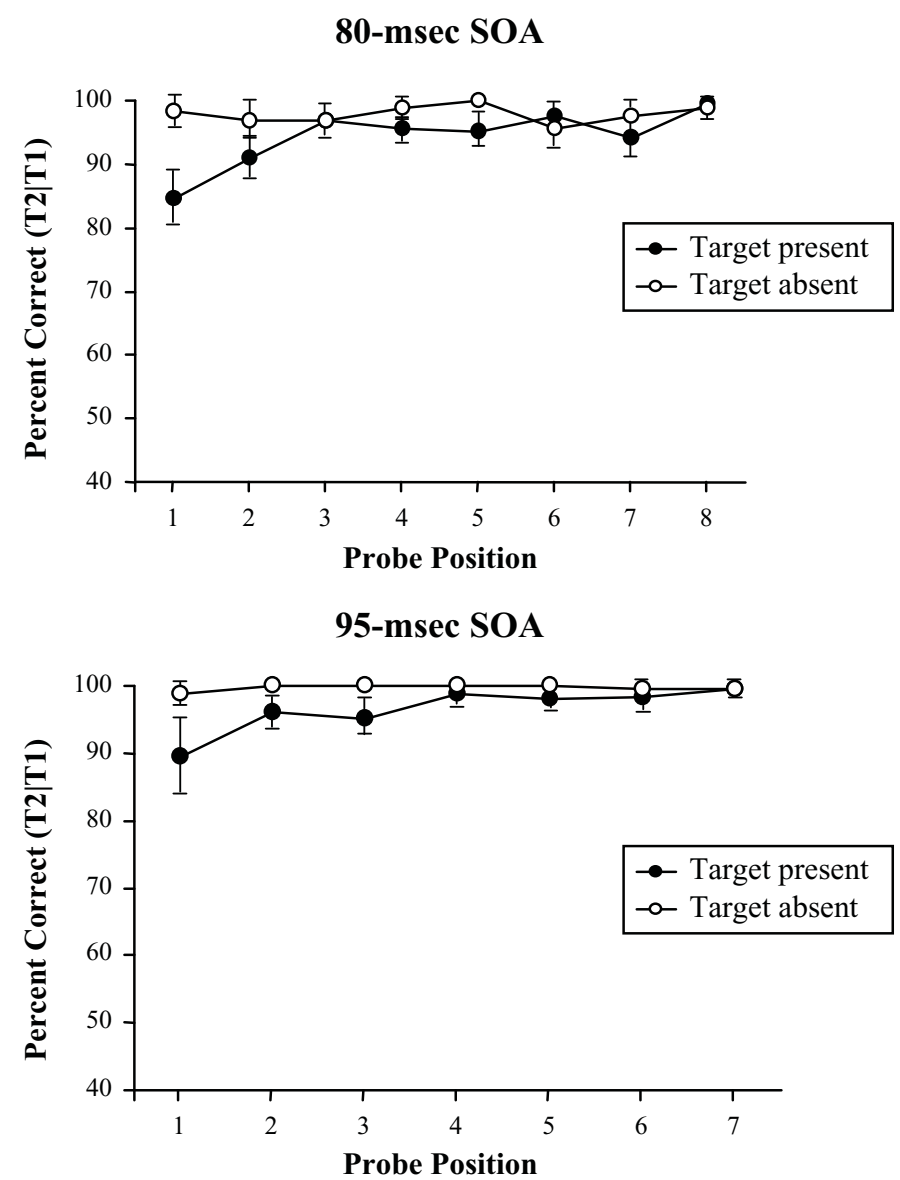

Figure 4. Probe performance in Experiment 4 as a function of SOA when all distractors following the probe were replaced by silence. Error bars represent \pm 1 standard error.

The magnitude of the auditory $\mathrm{AB}$ deficit was reduced substantially relative to the magnitude apparent when the single item immediately following the probe was replaced by silence (Experiments 2 and 3 ). This suggests that distractor sounds other than the one immediately following the probe can also overwrite the memorial representation of the probe. It is clear, however, that a very small auditory $\mathrm{AB}$ remains even when all distractor sounds following the probe are replaced by an extended silent period. Thus, the distractor sounds prior to the target and between the target and the probe may also play a small role in causing the auditory $\mathrm{AB}$. In theorizing about the visual $\mathrm{AB}$, Chun and Potter (1995) have suggested that a global effect of distractors has influence on the magnitude of the visual AB. According to this view, the presence of distractor items reduces discriminability of the target and the probe, and the time required to process the target is lengthened relative to when the target is presented in isolation. If processing of the probe is delayed until target processing is complete, then this delay could generate an auditory $A B$ due to an increased probability of decay of the probe representations. There might also be an effect of the distractors on the discriminability of the probe such that the quality of the memorial representation of the probe is reduced when it is presented in the context of other distractor sounds, and this also could increase the possibility of decay of the probe representation. Thus, when all distractors following the probe were eliminated in the present experiment, a small auditory AB still can be produced because of the decay of the probe representations. Such a view is consistent with our finding that the accuracy of the probe detection was higher in the 95- than in the 80-msec SOA sequences. It may be that the lengthened SOA $(95 \mathrm{msec})$ improved discriminability of the target and the probe relative to the 80 -msec SOA condition.

\section{GENERAL DISCUSSION}

The present study was conducted to examine the factors that affect the auditory $\mathrm{AB}$ as a means of moving toward a theoretical account of it. In Experiment 1, we examined whether the presence of distractors affected the magnitude of the $\mathrm{AB}$ and whether any resultant effect could be produced by sensory masking of the probe by the target. A significant auditory $\mathrm{AB}$ was apparent only when the target and the probe were presented in a sequence of distractor 
sounds. In addition, the magnitude of the auditory $\mathrm{AB}$ was significantly larger when the listeners were instructed to detect the target than when they were instructed to ignore it. The latter effect indicates that sensory masking of the probe by the target is not a principal cause of the auditory AB.

In Experiment 2, we examined the effect of deleting the item either immediately preceding or immediately following either the target or the probe. The results revealed that the magnitude of the $\mathrm{AB}$ was reduced only when the distractor immediately following the probe was deleted. Interestingly, in contrast with investigations of the visual $\mathrm{AB}$, eliminating the item following the target did not significantly influence the magnitude of the auditory $\mathrm{AB}$ in either experiment, although the difficulty of the target processing was reduced. In Experiment 3, we replicated the important effect of the postprobe distractor sound and the irrelevance of the posttarget distractor sounds for slower sequences with an SOA of $95 \mathrm{msec}$.

Finally, in Experiment 4, we deleted all of the distractor sounds following the probe in order to determine whether or not these items might also contribute to the auditory AB. Interestingly, we found that a small auditory $\mathrm{AB}$ remained even when no distractor sounds followed the probe. Because pretarget and posttarget distractors remained, this finding suggests that they may also play some small role in generating the auditory AB. However, the results of the four experiments we described above establish that the postprobe distractor sounds are especially important in generating an auditory AB.

\section{Auditory Attentional Blink and Lag-1 Sparing}

The probe processing deficit could be caused by a variety of different mechanisms. For example, the switching from searching for a target to searching for a probe could, in and of itself, create impairment in detecting a probe presented within the temporal period of this "taskswitching." Chun and Potter (2001; see also Potter, Chun, Banks, \& Muckenhoupt, 1998) proposed that "lag-1 sparing" may help identify a true AB. Lag-1 sparing occurs when the probe processing deficit at the +1 position is less than the maximum deficit. In other words, according to Chun and Potter (2001), the performance in detecting the probe should be a U-shaped function of probe position rather than a linear function for a true $\mathrm{AB}$. Chun and Potter (2001) suggested that if the probe processing deficit is attentional in nature, then the probe should be processed together with the target when the probe is presented in the +1 position, its processing would not be delayed, and only a minimal deficit would be apparent. Because Chun and Potter (2001; see also Potter et al., 1998) found no evidence that the auditory $\mathrm{AB}$ was consistent with this performance criterion, they argued that it resulted from task-switching. It is true that the present experimental results and the results of most other auditory studies (Arnell \& Jolicœur, 1999; Arnell \& Larson, 2002; Duncan et al., 1997; Mondor, 1998; but see Soto-Faraco \& Spence, 2002, for evidence of a U-shaped function) showed a gen- erally linear probe processing deficit. However, we do not agree that this pattern indicates that the auditory probe processing deficit is caused by task-switching because the difference between a linear and a U-shaped curve may simply provide an indication as to whether the item immediately following the target is processed along with it. Task-switching is just one of many possible explanations for why the probe is not processed together with the target. Our finding that replacement of the +1 distractor with a silent period has no effect on the auditory $\mathrm{AB}$ suggests that the +1 item is not processed together with the target, and this may be because the auditory system generally has better temporal resolution and poorer spatial resolution than does the visual system. Accordingly, when the probe is presented at the +1 position, the auditory system may be more likely than the visual system to process the target and the probe sequentially. It is true that U-shaped probe performance is more frequently reported in studies of the visual $\mathrm{AB}$ than in studies of the auditory $\mathrm{AB}$. It is interesting to note, however, that only about half of the studies of the visual AB report such a pattern (Visser, Bischof, \& Di Lollo, 1999). In brief, the U-shaped pattern is not essential to an $\mathrm{AB}$.

\section{Summary}

Taken together, the results of the four experiments we described above provide strong evidence that the auditory $A B$ is primarily attentional in nature and is neither the product of sensory masking of the probe by the target nor the product of task-switching between the target and the probe. Rather, it is clear that overwriting of the probe by postprobe distractor sounds is the major factor in generating the auditory $\mathrm{AB}$. In addition, it appears that a reduction in discriminability generated by all of the distractors presented in the sequence may also contribute to the auditory $\mathrm{AB}$. Whereas little evidence was obtained in favor of either the attentional gate model (see, e.g., Raymond et al., 1992) or the competition hypothesis (see, e.g., Shapiro et al., 1994), our results are consistent with a modified version of a two-stage model previously proposed to explain the visual AB (Chun \& Potter, 1995; Giesbrecht \& Di Lollo, 1998; Jolicœur, 1998). In this modified twostage model, listeners initially detect each sound in the sequence. However, to report the target and the probe successfully, the target items must be processed in a second stage (i.e., they must undergo short-term consolidation). Because the consolidation is a controlled, limited-capacity process, the target and the probe cannot be processed concurrently. Thus, the internal representation of the probe must be held in abeyance until target processing is complete. During this delay, the probe representation is subject to both passive decay and overwriting by the distractor sounds following it, and this directly impairs probe detection. Thus, the probe processing deficit is greatest when the probe is presented immediately following the target, because the delay is longest and the opportunity for decay or overwriting is greatest. 


\section{REFERENCES}

Arnell, K. M., \& Joliceleur, P. (1999). The attentional blink across stimulus modalities: Evidence for central processing limitations. Journal of Experimental Psychology: Human Perception \& Performance, 25, 630-648.

Arnell, K. M., \& Larson, J. M. (2002). Cross-modality attentional blinks without preparatory task-set switching. Psychonomic Bulletin \& Review, 9, 497-506.

Broadbent, D. E., \& Broadbent, M. H. P. (1987). From detection to identification: Response to multiple targets in rapid serial visual presentation. Perception \& Psychophysics, 42, 105-113.

Chun, M. M., \& PotTer, M. C. (1995). A two-stage model for multiple target detection in rapid serial visual presentation. Journal of Experimental Psychology: Human Perception \& Performance, 21, 109-127.

Chun, M. M., \& Potter, M. C. (2001). The attentional blink and task switching within and across modalities. In K. Shapiro (Ed.), The limits of attention: Temporal constraints on human information processing (pp. 20-35). Oxford: Oxford University Press.

Duncan, J., Martens, S., \& Ward, R. (1997). Restricted attentional capacity within but not between sensory modalities. Nature, $\mathbf{3 8 7}$, 808810.

Duncan, J., Ward, R., \& Shapiro, K. L. (1994). Direct measurement of attentional dwell time in human vision. Nature, 369, 313-315.

Folk, C. L., Leber, A. B., \& Egeth, H. E. (2002). Made you blink! Contingent attentional capture produces a spatial blink. Perception $\&$ Psychophysics, 64, 741-753.

Giesbrecht, B., Bischof, W. F., \& Kingstone, A. (2003). Visual masking during the attentional blink: Test of the object substitution hypothesis. Journal of Experimental Psychology: Human Perception \& Performance, 29, 238-258.

Giesbrecht, B., \& Di Lollo, V. (1998). Beyond the attentional blink: Visual masking by object substitution. Journal of Experimental Psychology: Human Perception \& Performance, 24, 1454-1466.

IsAaK, M. I., Shapiro, K. L., \& Martin, J. (1999). The attentional blink reflects retrieval competition among multiple rapid serial visual presentation items: Tests of an interference model. Journal of Experimental Psychology: Human Perception \& Performance, 25, 1774-1792.

JiAnG, Y., \& ChUn, M. M. (2001). The influence of temporal selection on spatial selection and distractor interference: An attentional blink study. Journal of Experimental Psychology: Human Perception \& Performance, 27, 664-679.

JolicEeUr, P. (1998). Modulation of the attentional blink by on-line response selection: Evidence from speeded and unspeeded Task $\mathrm{I}_{1}$ decisions. Memory \& Cognition, 26, 1014-1032.

Joliceeur, P., \& Dell'Acqua, R. (1998). The demonstration of shortterm consolidation. Cognitive Psychology, 36, 138-202.

Kawahara, J.-I., Di Lollo, V., \& Enns, J. T. (2001). Attentional requirements in visual detection and identification: Evidence from the attentional blink. Journal of Experimental Psychology: Human Perception \& Performance, 27, 969-984.

Kawahara, J.-I., Zuvic, S. M., Enns, J. T., \& Di Lollo, V. (2003).
Task switching mediates the attentional blink even without backward masking. Perception \& Psychophysics, 65, 339-351.

Kellie, F. J., \& Shapiro, K. L. (2004). Object file continuity predicts attentional blink magnitude. Perception \& Psychophysics, 66, 692712 .

Maxwell, S. E., \& Delaney, H. D. (2004). Designing experiments and analyzing data: A model comparison perspective (2nd ed.). Mahwah, NJ: Erlbaum.

Mondor, T. A. (1998). A transient processing deficit following selection of an auditory target. Psychonomic Bulletin \& Review, 5, 305-311.

Olson, I. R., Chun, M. M., \& Anderson, A. K. (2001). Effects of phonological length on the attentional blink for words. Journal of Experimental Psychology: Human Perception \& Performance, 27, 1116-1123.

Potter, M. C., Chun, M. M., Banks, B. S., \& Muckenhoupt, M. (1998). Two attentional deficits in serial target search: The visual attentional blink and an amodal task-switch deficit. Journal of Experimental Psychology: Learning, Memory, \& Cognition, 24, 979-992.

Potter, M. C., Staub, A., \& O'Connor, D. H. (2002). The time course of competition for attention: Attention is initially labile. Journal of Experimental Psychology: Human Perception \& Performance, 28, 1149-1162.

RAYMOND, J. E. (2003). New objects, not new features, trigger the attentional blink. Psychological Science, 14, 54-59.

Raymond, J. E., Shapiro, K. L., \& Arnell, K. M. (1992). Temporary suppression of visual processing in an RSVP task: An attentional blink? Journal of Experimental Psychology: Human Perception \& Performance, 18, 849-860.

Raymond, J. E., Shapiro, K. L., \& Arnell, K. M. (1995). Similarity determines the attentional blink. Journal of Experimental Psychology: Human Perception \& Performance, 21, 653-662.

Seiffert, A. E., \& Di Lollo, V. (1997). Low-level masking in the attentional blink. Journal of Experimental Psychology: Human Perception \& Performance, 23, 1061-1073.

Shapiro, K. L., Raymond, J. E., \& Arnell, K. M. (1994). Attention to visual pattern information produces the attentional blink in rapid serial visual presentation. Journal of Experimental Psychology: Human Perception \& Performance, 20, 357-371.

Soto-Faraco, S., \& Spence, C. J. (2002). Modality-specific auditory and visual temporal processing deficits. Quarterly Journal of Experimental Psychology, 55A, 23-40.

Tremblay, S., Vachon, F., \& Jones, D. M. (2005). Attentional and perceptual sources of the auditory attentional blink. Perception \& Psychophysics, 67, 195-208.

Visser, T. A. W., Bischof, W. F., \& Di Lollo, V. (1999). Attentional switching in spatial and nonspatial domains: Evidence from the attentional blink. Psychological Bulletin, 125, 458-469.

Ward, R., Duncan, J., \& Shapiro, K. (1996). The slow time-course of visual attention. Cognitive Psychology, 30, 79-109.

(Manuscript received July 27, 2004; revision accepted for publication April 29, 2005.) 